\title{
Medical Image of the Week: Lung Entrapment
}

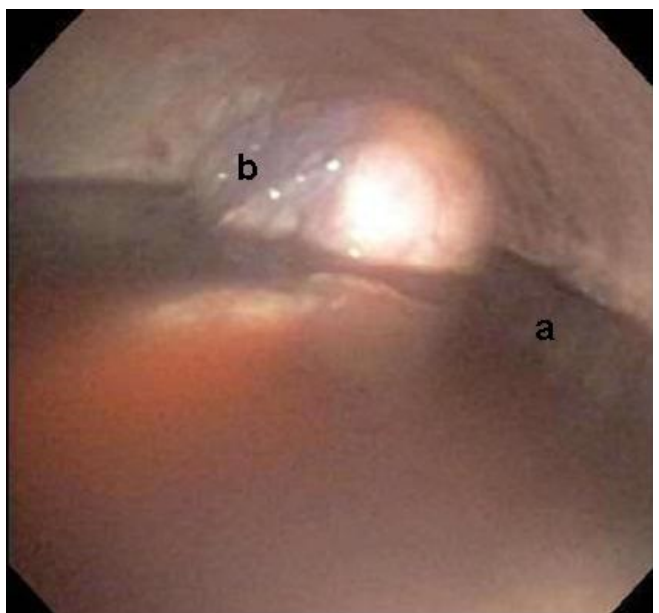

Figure 1. Pleural Fluid (a) and the collapsed left lung within the hemi-thorax (b).

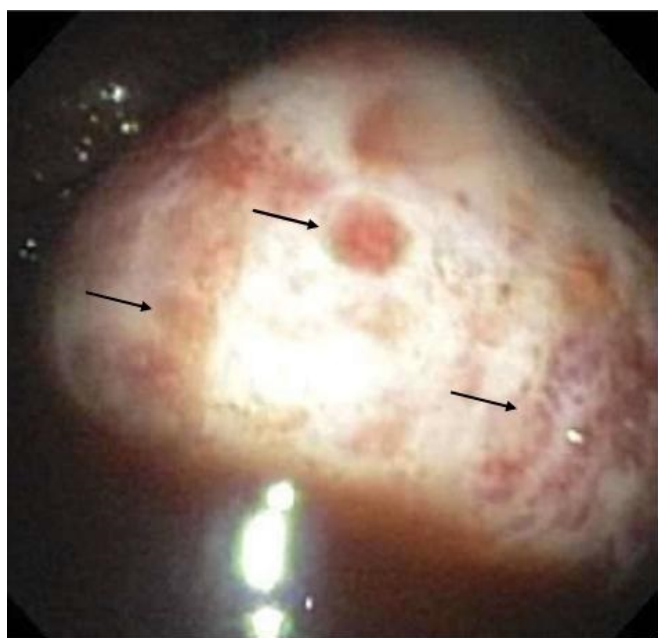

Figure 2. Malignant involvement of the visceral pleura (arrows)

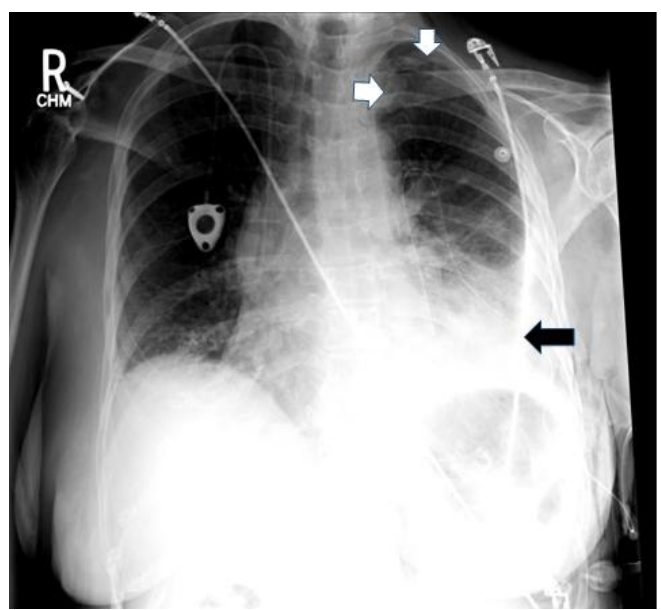

Figure 3. Persistent pneumothorax (white arrows) after several days of pleural catheter (black arrow) drainage. 
A 74-year-old woman with a history of breast cancer 10 years ago treated with lumpectomy and radiation presented for evaluation of shortness of breath. She was diagnosed with left sided pleural effusion which was recurrent requiring multiple thoracenteses. There was increased pleural fludeoxyglucose (FDG) uptake on PET-CT indicative of recurrent metastatic disease. She underwent a medical pleuroscopy since the pleural effusion analysis did not reveal malignant cells although the suspicion was high and tunneled pleural catheter placement as adjuvant chemotherapy was initiated. Figure 1 shows a pleurscopic view of the collapsed left lung and the effusion in the left hemi thorax. Figure 2 shows extensive involvement of the visceral pleura with metastatic disease preventing complete lung inflation. Figure 3 shows persistent pneumothorax-ex-vacuo despite pleural catheter placement confirming the diagnosis of entrapment.

Incomplete lung inflation can be due to pleural disease, endobronchial lesions or chronic telecasts.

Lung entrapment and trapped lung are related but distinct clinical entities (1). A trapped lung is a proper diagnosis when there is no active pleural disease however a fibrous peel has been formed due to a remote process and the mechanical effects of the pleura are the primary problem. Lung entrapment is used when incomplete lung inflation is secondary to visceral pleural peel secondary to active infection, inflammation or malignancy and the underlying process then becomes the primary problem.

The parietal pleural biopsies obtained during the pleuroscopy confirmed recurrent metastatic disease and the patient is currently undergoing chemotherapy.

Bhupinder Natt MD and James Knepler MD

Division of Pulmonary, Allergy, Critical Care and Sleep

University of Arizona Health Sciences,

Tucson, AZ USA

\section{Reference}

1. Huggins JT, Doelken P, Sahn SA. The unexpandable lung. F1000 Med Rep. 2010 Oct 21;2:77. [CrossRef] [PubMed] 